\title{
Erratum to: Outcome of Rehabilitation and Swallowing Therapy after Percutaneous Endoscopic Gastrostomy in Dysphagia Patients
}

\author{
Ezekiel Wong Toh Yoon $^{1} \cdot J_{u n}$ Hirao $^{2} \cdot$ Naoko Minoda $^{2}$
}

Published online: 24 August 2016

(c) Springer Science+Business Media New York 2016

\section{Erratum to: Dysphagia}

DOI 10.1007/s00455-016-9717-7

The original version of this article unfortunately contained a mistake. There are typos in the section "Results", last paragraph and the article title. The original article was also corrected.

The correct information is given below.

1. The standardized coefficient with $95 \%$ confidence interval for FIM score should be " $\beta$ 0.680, $95 \% \mathrm{CI}$ : 0.445 to $0.915 "$. The sentence should read as,

Finally, analysis of covariance showed that the presence of diabetes mellitus $(\beta-0.200,95 \%$ CI -0.398 to -0.003$)$ and respiratory disorders $(\beta$ $-0.259,95 \%$ CI -0.479 to -0.039$)$ were negatively associated with, and an increase in FIM score ( $\boldsymbol{\beta}$ 0.680, $95 \%$ CI 0.445 to 0.915 ) was positively associated with the changes in dysphagia grade (Table 7).

2. In title, the "After" should read as "after".

The online version of the original article can be found under doi:10.1007/s00455-016-9717-7.

\section{Ezekiel Wong Toh Yoon}

easybs@hotmail.com

1 Department of Internal Medicine, Hiroshima Kyoritsu Hospital, 2-20-20 Nakasu Asaminami-ku, Hiroshima, Japan

2 Department of Rehabilitation, Hiroshima Kyoritsu Hospital, 2-20-20 Nakasu Asaminami-ku, Hiroshima, Japan 